\title{
Projection-based Iterative Learning Control for Ball-screw-driven Stage with Consideration of Rolling Friction Compensation
}

\author{
Takumi Hayashi*a) $^{*}$ Student Member, \\ Hiroshi Fujimoto*

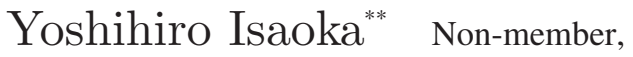 \\ Yuki Terada** \\ Senior Member \\ Non-member
}

(Manuscript received May 9, 2019, revised Nov. 14, 2019)

\begin{abstract}
Ball-screw-driven stages are feed systems that are widely used in industrial equipment such as numerically controlled machine tools. They require precise position control; however, rolling friction in the ball-screw mechanism deteriorates its control performance. Therefore, for precise control of ball-screw-driven stages, rolling friction must be compensated. Iterative learning control (ILC) is an effective method of friction compensation. However, industrial applications of ILC are limited because of its vulnerability to task variation, such as variation in position reference. In conventional studies, projection-based ILC employing basis functions has been proposed to deal with multiple tasks. In this paper, we propose basis functions for position control of ball-screw-driven stages based on their physical association with rolling friction. Simulations and experiments validate the effectiveness of our proposal.
\end{abstract}

Keywords: iterative learning control, projection-based iterative learning control, ball-screw-driven stage, rolling friction

\section{Introduction}

Ball-screw-driven stages are feed systems that convert rotational motion of motor into translational motion of stage. Figure 1 shows their schematic view. They are widely used in industrial equipment such as numerically controlled (NC) machine tools. In NC machine tools, precise machining is required for high production quality. Therefore, precise position control of ball-screw-driven stages is necessary.

However, this is difficult to achieve due to rolling friction. Rolling friction can be characterized into two regions, "region 1" and "region 2", as shown in Fig. 2. In region 1, rolling friction is nonlinear and depends on displacement from the velocity reversal. In region 2 , rolling friction is almost constant demonstrating Coulomb friction $T_{c}$. A large tracking error arises near the velocity reversal because of this friction.

Various studies have been conducted to compensate for rolling friction. The compensation methods are classified into two types: model-based methods and learning-based methods. In model-based methods, various models of rolling friction have been proposed, e.g., LuGre model ${ }^{(1)}$, generalized Maxwell-slip model ${ }^{(2)}$, rheology-based model ${ }^{(3)}$, variable natural length spring model ${ }^{(4)}$, and data-based model ${ }^{(5)}$. These model-based methods require the precise measurement of rolling friction, and friction models are tuned based on the measurement data. Rolling friction can be compensated if these models are well-tuned. However, tuning these models is time-consuming, and rolling friction is not compensated

\footnotetext{
a) Correspondence to: Takumi Hayashi. E-mail: hayashi.takumi18 @ae.k.u-tokyo.ac.jp

* The University of Tokyo

5-1-5, Kashiwanoha, Kashiwa, Chiba 277-8561, Japan

** DMG MORI CO., LTD.

362, Idono, Yamatokoriyama, Nara 639-1183, Japan
}

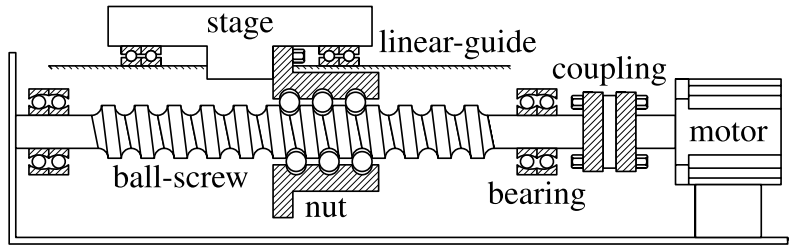

Fig. 1. Schematic view of ball-screw-driven stages

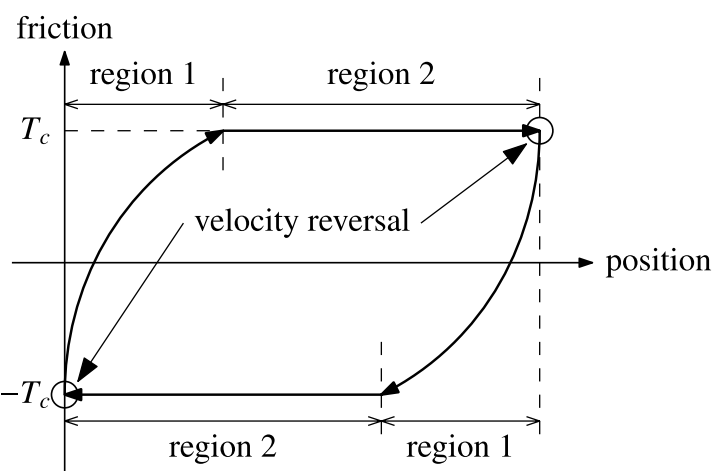

Fig. 2. Characteristics of rolling friction of ball-screwdriven stages

because it depends on the environment and operating conditions (e.g., mass variation of the workpiece on the stage ${ }^{(6)}$ ).

This study adopts the learning-based methods ${ }^{(7)}$. In learning-based methods, rolling friction is compensated by repeating the same task. Iterative learning control (ILC) ${ }^{(8)(9)}$ is one of the learning-based control methods. Standard ILC (S-ILC) has a disadvantage that relearning is required after the task is changed. To overcome this problem, studies on ILC using basis functions called projection-based ILC (PILC) have been conducted ${ }^{(10)(11)}$. In P-ILC, basis functions are introduced to deal with multiple tasks. In terms of the 
parameter estimation using basis functions, P-ILC is similar to typical adaptive control ${ }^{(12)(13)}$, with the difference being the timing of the parameter estimation ${ }^{(10)}$. Typical adaptive control updates the parameters at each sampling time using online estimation algorithms, such as recursive least squares method. On the other hand, P-ILC estimates the parameters during the interval between trials by using the complete information of one trial. Therefore, the persistent excitation condition can be easily confirmed and satisfied in P-ILC. Also, it can enhance parameter estimation because the number of available data in the parameter estimation is larger than adaptive control.

However, rolling friction compensation was not considered in these studies. In another study ${ }^{(14)}$, P-ILC was used for system and friction identifications of a ball-screw-driven stage, but it did not consider the displacement dependency of the friction.

Therefore, in this study, to achieve precise control of ballscrew-driven stages considering rolling friction compensation, P-ILC using new basis functions for rolling friction compensation is proposed. Its effectiveness is demonstrated through simulations and experiments.

This paper is organized as follows: First, experimental setup is described in Section 2; S-ILC and P-ILC are introduced in Section 3 and Section 4; simulations and experiments are discussed in Section 5 and Section 6 to demonstrate the effectiveness of our proposal; and finally, the conclusion and future work are presented in Section 7.

\section{Experimental Setup}

2.1 Modeling The experimental setup used in this study is depicted in Fig. 3. This experimental setup has two axes, $x$-axis and $y$-axis. Only the $x$-axis is used in this study. Figure 4 shows the frequency response data from the motor current $i[\mathrm{~A}]$ to the stage position $x[\mathrm{~m}]$ of the $x$-axis measured by frequency domain identification ${ }^{(15)}$. In this study, we only consider the rigid mode, and the nominal model of this experimental setup is expressed as

$$
P_{n}(s)=\frac{x}{i}=\frac{R K_{T}}{J_{n} s^{2}+D_{n} s}
$$

Parameters in (1) are shown in Table 1.

2.2 Rolling Friction Rolling friction of the experimental setup is shown in Fig. 5. This data is measured by ultra-low speed examination and disturbance observer to suppress the influence of modeling error. According to Fig. 5, the range of region 1 and Coulomb friction $T_{c}$ in Fig. 2 are identified to be $10 \mu \mathrm{m}$ and $3.2 \mathrm{Nm}$, respectively.

Note that the "simulation model" in Fig. 5 is only used in the simulation referred in Section 5 and not used for any controller design.

\section{Standard Iterative Learning Control}

3.1 Lifted System Representation ILC is one of the learning-based control methods, which becomes effective when the same task is repeated. By repeating the same task and updating feedforward input from the previous trial's error, tracking error is gradually suppressed.

First, lifted system representation ${ }^{(8)}$ is introduced. A sequential signal of ILC's one trial $\boldsymbol{w}$ is represented as

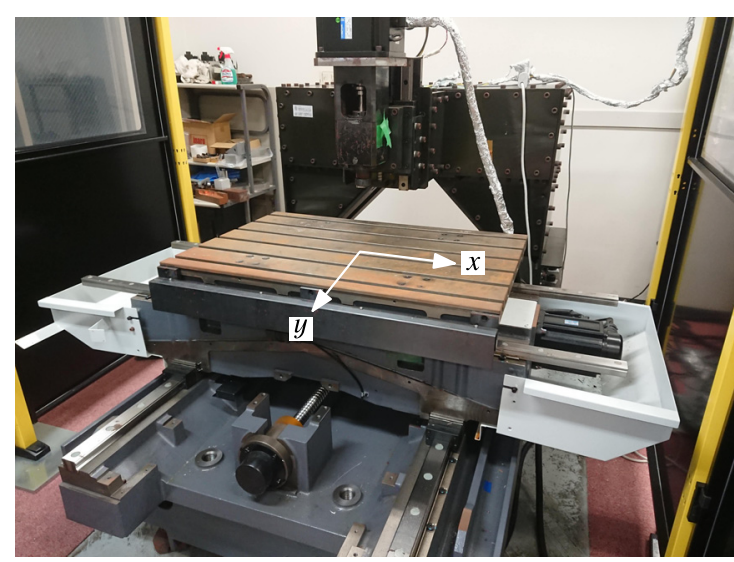

Fig. 3. Picture of the experimental setup
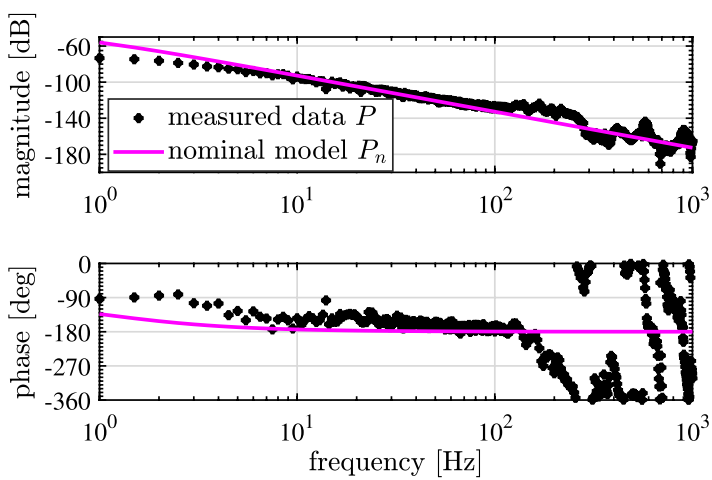

Fig. 4. Frequency response from motor current $i$ [A] to stage position $x[\mathrm{~m}]$ of the $x$-axis of the experimental setup

Table 1. Parameters of the experimental setup

\begin{tabular}{cc}
\hline Nominal inertia $J_{n}$ & $0.015 \mathrm{kgm}^{2}$ \\
\hline Nominal viscosity coefficient $D_{n}$ & $0.1 \mathrm{~N} \mathrm{~m} \mathrm{~s} \mathrm{rad}^{-1}$ \\
\hline Torque constant $K_{T}$ & $0.715 \mathrm{~N} \mathrm{~m} \mathrm{~A}^{-1}$ \\
\hline Ball-screw's lead $R$ & $1.91 \mathrm{~mm} \mathrm{rad}^{-1}$ \\
\hline
\end{tabular}

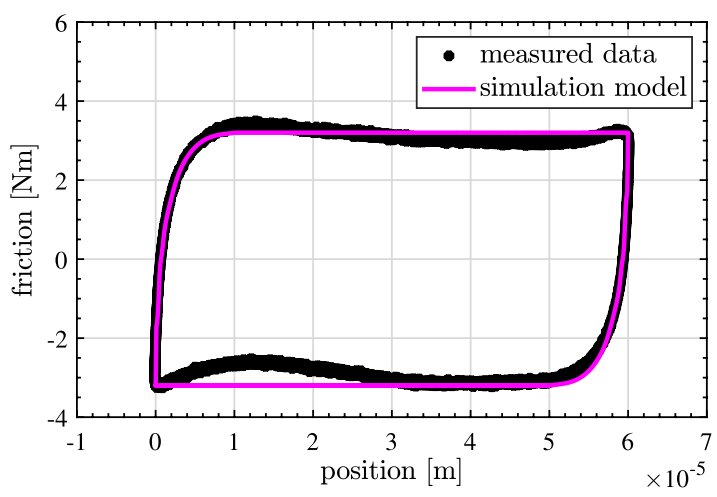

Fig. 5. Rolling friction of the experimental setup

$$
\begin{aligned}
& \boldsymbol{w}=\left[\begin{array}{llll}
w[0] & w[1] & \cdots & w[N]
\end{array}\right]^{\top} \in \mathbb{R}^{N+1}, \cdots \cdots \\
& w[k]=w\left(k T_{s}\right), \cdots \cdots \cdots \cdots \cdots \cdots \cdots \cdots \cdots \cdots
\end{aligned}
$$

where $N+1$ is the length of one trial and $T_{S}$ is the sampling time. The convolution matrix $\mathcal{P}$ of a discrete-time linear time-invariant system $P$ is represented as follows: 


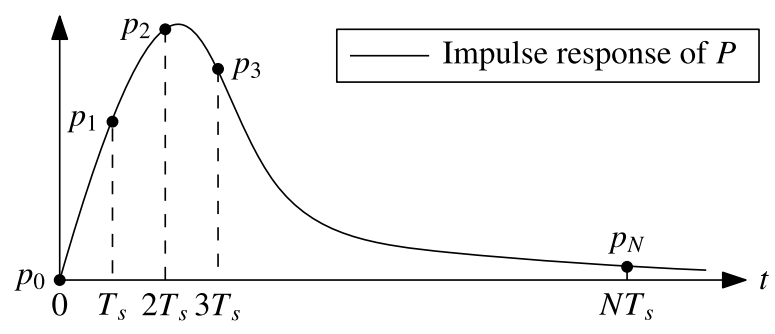

Fig. 6. Impulse response coefficient of a discrete-time linear time-invariant system $P$

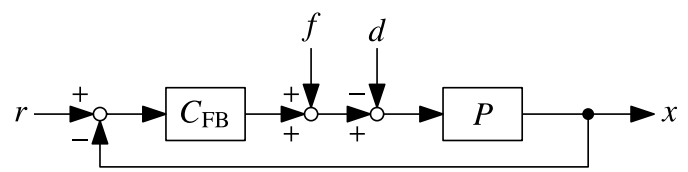

Fig. 7. Block diagram of two-degree-of-freedom control system. $r$ : position reference, $x$ : position output, $P$ : linear time-invariant system, $C_{\mathrm{FB}}$ : feedback controller, $f$ : feedforward input, $d$ : disturbance (rolling friction)

$$
\mathcal{P}=\left[\begin{array}{cccc}
p_{0} & 0 & \cdots & 0 \\
p_{1} & p_{0} & \cdots & 0 \\
\vdots & \vdots & \ddots & 0 \\
p_{N} & p_{N-1} & \cdots & p_{0}
\end{array}\right] \in \mathbb{R}^{(N+1) \times(N+1)}, \cdots \cdots
$$

where $p_{i}(i=0,1, \ldots, N)$ denotes the impulse response coefficient, as shown in Fig. 6. By using lifted system representation, the relation between the input $i$ and the output $x$ of the system $P$ is given by

$$
\begin{aligned}
& \boldsymbol{x}=\mathcal{P} \boldsymbol{i}, \cdots \cdots \cdots \cdots \cdots \cdots \cdots \\
& \boldsymbol{i}=\left[\begin{array}{llll}
i[0] & i[1] & \cdots & i[N]
\end{array}\right]^{\top}, \\
& \boldsymbol{x}=\left[\begin{array}{llll}
x[0] & x[1] & \cdots & x[N]
\end{array}\right]^{\top}
\end{aligned}
$$

3.2 Tracking Error Suppression by Standard Iterative Learning Control Two-degree-of-freedom control system as shown in Fig. 7 is considered. In Fig. 7, tracking error $e$ is given by

$$
e=r-x=S r-S P(f-d),
$$

where $S$ is a sensitivity function:

$$
S=\frac{1}{1+C_{\mathrm{FB}} P}
$$

In the $j$ th trial of ILC, by using lifted system representation, (5) can be rewritten as

$$
\boldsymbol{e}_{j}=\mathcal{S} \boldsymbol{r}_{j}-\mathcal{S P}\left(\boldsymbol{f}_{j}-\boldsymbol{d}_{j}\right)
$$

Here, subscript $j$ denotes ILC's trial number. In ILC, next trial's feedforward input $\boldsymbol{f}_{j+1}$ is generated from $\boldsymbol{f}_{j}$ and $\boldsymbol{e}_{j}$ as follows:

$$
\boldsymbol{f}_{j+1}=Q\left(\boldsymbol{f}_{j}+\mathcal{L} \boldsymbol{e}_{j}\right)
$$

$\mathcal{L}$ and $Q$ are the convolution matrices corresponding to the learning filter $L(z)$ and robust filter $Q(z)$, respectively. Here, $z$ denotes $\mathrm{e}^{s T_{s}}$.

From (7) and (8), next trial's tracking error $\boldsymbol{e}_{j+1}$ is expressed as

$$
\begin{aligned}
\boldsymbol{e}_{j+1} & =\mathcal{S} \boldsymbol{r}_{j+1}-\mathcal{S P}\left(\boldsymbol{f}_{j+1}-\boldsymbol{d}_{j+1}\right) \\
& =\boldsymbol{Q}(\boldsymbol{I}-\mathcal{S P} \mathcal{L}) \boldsymbol{e}_{j}+(\boldsymbol{I}-\boldsymbol{Q})(\mathcal{S} \boldsymbol{r}-\mathcal{S P} \boldsymbol{d})
\end{aligned}
$$

with the assumption that the position reference $r$ and the disturbance $\boldsymbol{d}$ are the same in all the trials:

$$
\begin{aligned}
\boldsymbol{r} & :=\boldsymbol{r}_{1}=\boldsymbol{r}_{2}=\cdots, \\
\boldsymbol{d} & :=\boldsymbol{d}_{1}=\boldsymbol{d}_{2}=\cdots
\end{aligned}
$$

From the recurrence formula (9), tracking error of ILC monotonically decreases if (11) is satisfied.

$$
\bar{\sigma}(\boldsymbol{Q}(\boldsymbol{I}-\mathcal{S P} \mathcal{L}))<1 \cdots
$$

Here, $\bar{\sigma}(\boldsymbol{A})$ denotes the maximum singular value of matrix A.

Some design methods of the two filters, $L$ and $Q$, exist $^{(8)}$, and in this study, $L$ and $Q$ are designed based on frequency response data. In frequency domain approach, ILC's monotonic convergence condition (11) denotes that gain of $Q(1-S L P)$ is less than 1 for all frequencies.

$$
\max _{\omega}\left|Q\left(\mathrm{e}^{\mathrm{j} \omega}\right)\left(1-S\left(\mathrm{e}^{\mathrm{j} \omega}\right) L\left(\mathrm{e}^{\mathrm{j} \omega}\right) P\left(\mathrm{e}^{\mathrm{j} \omega}\right)\right)\right|<1 . \cdots
$$

\section{Projection-based Iterative Learning Control for Multiple Tasks}

According to the previous section, S-ILC is an effective method when the position reference $\boldsymbol{r}$ is precisely repeated, but it has a disadvantage that relearning is required after the position reference changes. In order to overcome this problem, studies on P-ILC have been conducted.

In the $j$ th trial of P-ILC, the feedforward input $\boldsymbol{f}_{j}^{p}$ is parameterized as follows:

$$
\begin{gathered}
\boldsymbol{f}_{j}^{p}=\boldsymbol{\Psi}\left(\boldsymbol{r}_{j}\right) \boldsymbol{\theta}_{j}, \cdots \\
\boldsymbol{\Psi}\left(\boldsymbol{r}_{j}\right) \in \mathbb{R}^{(N+1) \times n_{\theta}}, \\
\boldsymbol{\theta}_{j} \in \mathbb{R}^{n_{\theta}}, \ldots \ldots \ldots
\end{gathered}
$$

where $\boldsymbol{\Psi}\left(\boldsymbol{r}_{j}\right)$ is the basis functions depending on the $j$ th trial's position reference $\boldsymbol{r}_{j}$, while $\boldsymbol{\theta}_{j}$ is the parameters independent of $\boldsymbol{r}_{j}$ but depending on the plant $P$. Superscript $p$ implies "P-ILC".

\subsection{Selection of Basis Functions}

4.1.1 Conventional Basis Functions From (5), when no disturbance exists $(d=0)$, feedforward input $f$ to follow the position output $x$ to the position reference $r$ is expressed as

$$
f=P^{-1} r \Rightarrow e=0
$$

Considering the ball-screw-driven stages' transfer function $P=R K_{T} /\left(J s^{2}+D s\right)$, (14) can be rewritten as follows:

$$
f=\frac{J}{R K_{T}} \ddot{r}+\frac{D}{R K_{T}} \dot{r} .
$$

Therefore, the basis functions $\boldsymbol{\Psi}\left(\boldsymbol{r}_{j}\right)$ and the parameters $\boldsymbol{\theta}_{j}$ of the $j$ th trial are determined as follows:

$$
\boldsymbol{\Psi}\left(\boldsymbol{r}_{j}\right)=\left[\begin{array}{cc}
\ddot{r}[0] & \dot{r}[0] \\
\ddot{r}[1] & \dot{r}[1] \\
\vdots & \vdots \\
\ddot{r}[N] & \dot{r}[N]
\end{array}\right],
$$




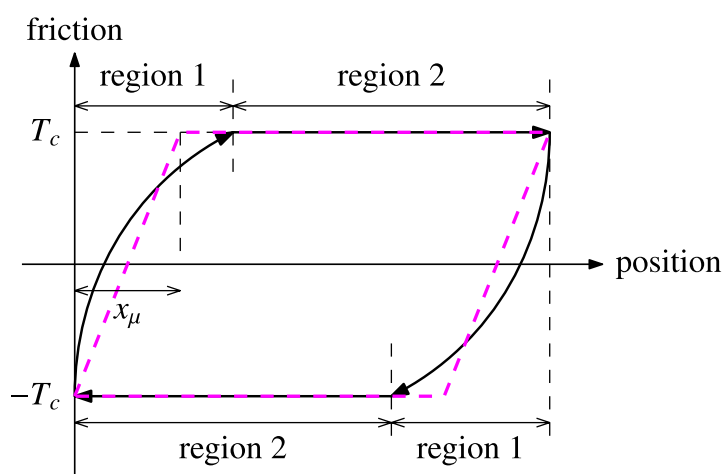

Fig. 8. Real characteristics (black solid line) and approximation (magenta dashed line) of rolling friction

$$
\boldsymbol{\theta}_{j}=\left[\begin{array}{ll}
J / R K_{T} & D / R K_{T}
\end{array}\right]^{\top} .
$$

In P-ILC, the plant parameters $J / R K_{T}$ and $D / R K_{T}$ are estimated by using S-ILC in each trial.

4.1.2 Proposed Basis Functions for Rolling Friction Compensation For precise control of ball-screw-driven stages, rolling friction should be compensated. To consider rolling friction in P-ILC and make the optimization problem easy in the next subsection, the rolling friction is approximated as shown in Fig. 8. The approximation model of rolling friction $d[\mathrm{Nm}]$ is expressed as

$$
\begin{aligned}
d(t) & \approx \operatorname{sign}(\dot{x}(t)) \cdot \min \left(\frac{2 T_{c}}{x_{\mu}} x_{r}(t)-T_{c}, T_{c}\right) \\
& =T_{c} \cdot \operatorname{sign}(\dot{x}(t)) \min \left(\frac{2}{x_{\mu}} x_{r}(t)-1,1\right) \\
& =T_{c} \cdot b_{\mathrm{rf}}(t), \ldots \ldots \ldots \ldots \ldots \ldots \ldots \ldots \ldots \ldots \ldots \ldots
\end{aligned}
$$

where

$$
b_{\mathrm{rf}}(t):=\operatorname{sign}(\dot{x}(t)) \min \left(\frac{2}{x_{\mu}} x_{r}(t)-1,1\right) \ldots \ldots
$$

Here, $x_{\mu}$ is a free parameter and $x_{r}$ is a displacement from the velocity reversal. In this study, $x_{\mu}$ is fixed. Therefore, it is assumed that only $T_{c}$ is variable. In the proposed P-ILC, $b_{\mathrm{rf}}(t)$ is a part of $\boldsymbol{\Psi}\left(\boldsymbol{r}_{j}\right)$, and Coulomb friction $T_{c}$ is a part of $\boldsymbol{\theta}_{j}$.

From the above discussion, the proposed basis functions $\boldsymbol{\Psi}\left(\boldsymbol{r}_{j}\right)$ and the parameters $\boldsymbol{\theta}_{j}$ are determined as follows:

$$
\begin{aligned}
& \boldsymbol{\Psi}\left(\boldsymbol{r}_{j}\right)=\left[\begin{array}{ccc}
\ddot{r}[0] & \dot{r}[0] & b_{\mathrm{rf}}[0] \\
\ddot{r}[1] & \dot{r}[1] & b_{\mathrm{rf}}[1] \\
\vdots & \vdots & \vdots \\
\ddot{r}[N] & \dot{r}[N] & b_{\mathrm{rf}}[N]
\end{array}\right], \\
& \boldsymbol{\theta}_{j}=\left[\begin{array}{lll}
J / R K_{T} & D / R K_{T} & T_{c} / K_{T}
\end{array}\right]^{\top}
\end{aligned}
$$

The displacement $x_{r}[k]$ is required to calculate $b_{\mathrm{rf}}[k]$ in the basis functions. In this study, $x_{r}[k]$ is calculated from the position reference.

4.2 Parameter Estimation To estimate parameters $\boldsymbol{\theta}_{j+1}$ for the $(j+1)$ th trial of P-ILC, an optimization problem is solved. According to $(7)$, tracking error of the $(j+1)$ th trial is expressed as follows:

$$
\boldsymbol{e}_{j+1}=\mathcal{S} \boldsymbol{r}_{j+1}-\mathcal{S P}\left(\boldsymbol{f}_{j+1}-\boldsymbol{d}_{j+1}\right)
$$

By assuming $\boldsymbol{r}_{j+1}=\boldsymbol{r}_{j}$ and $\boldsymbol{d}_{j+1}=\boldsymbol{d}_{j}, \boldsymbol{e}_{j+1}$ can be predicted as (21) from (7) and (20) by using nominal plant before the $(j+1)$ th trial is done.

$$
\hat{\boldsymbol{e}}_{j+1}=\boldsymbol{e}_{j}-\mathcal{S}_{n} \mathcal{P}_{n}\left(\boldsymbol{f}_{j+1}-\boldsymbol{f}_{j}\right),
$$

where $\hat{\boldsymbol{e}}_{j+1}$ is the predicted tracking error and subscript $n$ denotes the nominal plant's value. In the same way, the predicted tracking error $\hat{\boldsymbol{e}}_{j+1}^{p}$ of P-ILC's $(j+1)$ th trial is given by

$$
\hat{\boldsymbol{e}}_{j+1}^{p}=\boldsymbol{e}_{j}-\mathcal{S}_{n} \mathcal{P}_{n}\left(\boldsymbol{\Psi}\left(\boldsymbol{r}_{j}\right) \boldsymbol{\theta}_{j+1}-\boldsymbol{f}_{j}\right)
$$

Here, $\boldsymbol{\Psi}\left(\boldsymbol{r}_{j}\right) \boldsymbol{\theta}_{j+1}$ is the feedforward input calculated when $\boldsymbol{r}_{j+1}=\boldsymbol{r}_{j}$ and $\boldsymbol{d}_{j+1}=\boldsymbol{d}_{j}$.

In this study, the following optimization problem

$$
\begin{aligned}
& \min _{\boldsymbol{\theta}_{j+1}}\left\|\hat{\boldsymbol{e}}_{j+1}-\hat{\boldsymbol{e}}_{j+1}^{p}\right\|_{2} \ldots \ldots \ldots \ldots \ldots \ldots \ldots \ldots \\
& \Rightarrow \min _{\boldsymbol{\theta}_{j+1}}\left\|\mathcal{S}_{n} \boldsymbol{P}_{n} \boldsymbol{f}_{j+1}-\mathcal{S}_{n} \boldsymbol{P}_{n} \boldsymbol{\Psi}\left(\boldsymbol{r}_{j}\right) \boldsymbol{\theta}_{j+1}\right\|_{2}
\end{aligned}
$$

is solved by the least squares method to determine $\boldsymbol{\theta}_{j+1}{ }^{(10)}$. Note that the unique analytical solution of $\boldsymbol{\theta}_{j+1}$ can be obtained under the assumption that the matrix $\left(\mathcal{S}_{n} \mathcal{P}_{n} \boldsymbol{\Psi}\left(\boldsymbol{r}_{j}\right)\right)^{\top}$ $\mathcal{S}_{n} \mathcal{P}_{n} \boldsymbol{\Psi}\left(\boldsymbol{r}_{j}\right)$ is nonsingular, i.e., the position reference $\boldsymbol{r}_{j}$ is a persistently-exciting signal.

Finally, the position reference is updated to $\boldsymbol{r}_{j+1}$ and feedforward input is calculated as

$$
\boldsymbol{f}_{j+1}^{p}=\boldsymbol{\Psi}\left(\boldsymbol{r}_{j+1}\right) \boldsymbol{\theta}_{j+1}
$$

Then, $(j+1)$ th trial is done.

\section{Simulation}

To verify the effectiveness of the proposed method, two types of simulations are conducted: Case 1 and Case 2. Here are the conventional and proposed methods:

- Conventional method 1 (S-ILC): S-ILC without any basis functions.

- Conventional method 2 (Conv. P-ILC): P-ILC using the conventional basis functions (16).

- Proposed method (Prop. P-ILC): P-ILC using the proposed basis functions (19).

Sampling time $T_{s}$ is set as $1 \mathrm{~ms}$.

\subsection{Conditions}

5.1.1 Plant In the simulations, the nominal and simulation plants are the same as shown in Fig. 4. Therefore, modeling error is not considered in the simulations.

Rolling friction used in the simulations is shown in Fig. 5. The range of the region showing nonlinearity is $10 \mu \mathrm{m}$ and Coulomb Friction $T_{c}$ is $3.2 \mathrm{Nm}$.

5.1.2 Feedback Controller Feedback controller $C_{\mathrm{FB}}$ is a proportional-integral-differential controller designed to have $30 \mathrm{~Hz}$ closed-loop multiple poles by the pole placement method. It is discretized by the Tustin transformation with sampling time $T_{s}=1 \mathrm{~ms}$.

5.1.3 Learning Filter and Robust Filter The learning filter $L$ is designed as a zero phase error tracking controller ${ }^{(16)}$ of the closed-loop model $S_{n} P_{n}$. In addition, the robust filter $Q$ is a zero-phase low-pass filter (25). 


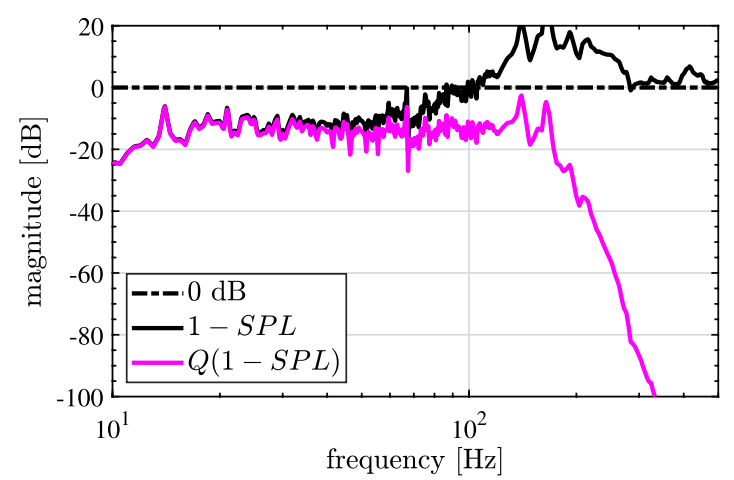

Fig. 9. Bode diagram of $1-S L P$ and $Q(1-S L P)$. $N_{Q}$ in (25) is set as 16

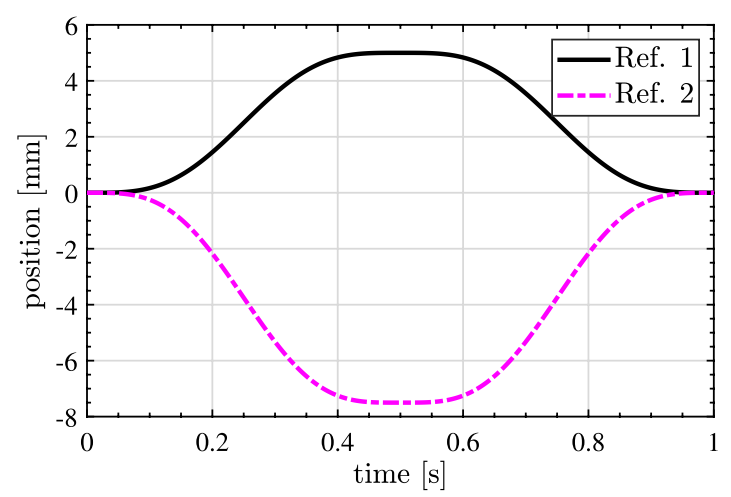

Fig. 10. Position references (Case 1)

$$
Q(z)=\left(\frac{z+2+z^{-1}}{4}\right)^{N_{Q}},
$$

where $N_{Q}$ is the order of $Q(z)$. $Q(z)$ is realized by $Q_{r}(z)$ with $N_{Q}$ samples delay compensation of the memory.

$$
Q_{r}(z)=Q(z) \cdot z^{-N_{Q}} \ldots \ldots \ldots \ldots \ldots \ldots \ldots \ldots \ldots
$$

$N_{Q}$ is set as 16 to satisfy ILC's convergence condition in the frequency domain (12). Figure 9 shows the gain of $1-S L P$ and $Q(1-S L P)$, and (12) is satisfied when $N_{Q}=16$.

5.2 Results (Case 1) Figure 10 shows the two types of position references used in simulations (Case 1). From the position references shown in Fig. 10, the basis functions of P-ILC are given as Fig. 11. Here, $x_{\mu}$ is set as $5 \mu \mathrm{m}$. This study considers the situation that the states of the system and rolling friction are initialized during the interval between trials. The initial value of the rolling friction in all the trials is considered $0 \mathrm{Nm}$. Therefore, the basis function $b_{\mathrm{rf}}(t)$ starts from 0 at $t=0 \mathrm{~s}$, see Fig. 11(c). First, "Ref. 1" is used until the 5 th trial of ILC. From the 6th trial, the position reference changes to "Ref. 2", to compare S-ILC and P-ILC from the perspective of robustness to the position reference variation.

The simulation results are shown in Fig. 12, demonstrating the effectiveness of the proposed P-ILC. Figures 12(a) and 12(b) show the Root-Mean-Square (RMS) error and maximum error of the $j$ th trial, respectively. Figures 12(c)-12(f) show the tracking error and feedforward input of one trial before/after the position reference variation. In addition, Figs. 12(g)-12(i) show the estimated parameters in the conventional and proposed P-ILC. Here, $\hat{\bullet}$ denotes the estimated parameter. Figures $12(\mathrm{j})$ and $12(\mathrm{k})$ compare the real value and the compensation of rolling friction.
5.2.1 Comparison of Conventional and Proposed P-

ILC According to Fig. 12, it can be said that the tracking error drastically decreases by using the basis functions (19). Figures 12(g)-12(i) show that all of the estimated parameters in the proposed P-ILC converge to values close to the actual values, while the estimated parameters in the conventional P-ILC do not match the actual values. By adding a new basis function for rolling friction compensation, the plant parameters can be estimated correctly. Figures 12(j) and $12(\mathrm{k})$ show that rolling friction is compensated well by the proposed P-ILC. The effectiveness of the proposed P-ILC is demonstrated.

5.2.2 Comparison of S-ILC and Proposed P-ILC

According to the tracking error and feedforward input after the position reference variation, shown in Figs. 12(d) and 12(f), it can be said that the proposed P-ILC is robust to position reference variation, while S-ILC cannot deal with the position reference variation. However, S-ILC is better from the viewpoint of tracking error after learning precisely repeated position reference, refer to the tracking error of the 5th trial shown in Figs. 12(a), 12(b), and 12(c). The reasons for a larger tracking error after finishing learning by the proposed P-ILC can be considered as follows:

- Difference between the actual plant parameters and the estimated parameters in the proposed P-ILC: Especially in terms of Coulomb friction $T_{c}$, the estimated value is smaller than the actual value. It can be considered that this difference causes a larger tracking error in the proposed P-ILC.

- Approximation of rolling friction: To consider rolling friction in P-ILC, it is approximated as shown in Fig. 8. Therefore, a difference exists between the actual rolling friction and its approximation model used in the proposed P-ILC. This causes a larger tracking error around the velocity reversal of the 5th trial, see Fig. 12(c).

5.3 Results (Case 2) To verify the effectiveness of the proposed P-ILC when the target motion is small and when the frequency components of the position reference vary, other simulations (Case 2) are conducted. In these simulations, the position references as shown in Fig. 13(a) are used. Until the 5th trial, "Ref. 3" is used, while "Ref. 4" is used from the 6th trial. Figures 13(b) and 13(c) show the convergence of the tracking error, which are similar to those of simulations (Case 1). From these simulations, the effectiveness of the proposed P-ILC can be verified.

\section{Experiment}

6.1 Conditions Experiments are conducted to verify the effectiveness of the proposed P-ILC. Experimental conditions are the same as those of simulations (Case 1).

6.2 Results Figure 14 shows the experimental results. Figures 14(a) and 14(b) show RMS error and maximum error of the $j$ th trial, respectively. Figures 14(c)-14(f) show the tracking error and feedforward input of one trial before/after the position reference variation. Also, Figs. 14(g)-14(i) show the estimated parameters in the conventional and proposed P-ILC.

The experimental results show the same tendency as those of the simulation, i.e., the proposed P-ILC is robust to the position reference variation and achieves precise control. How- 


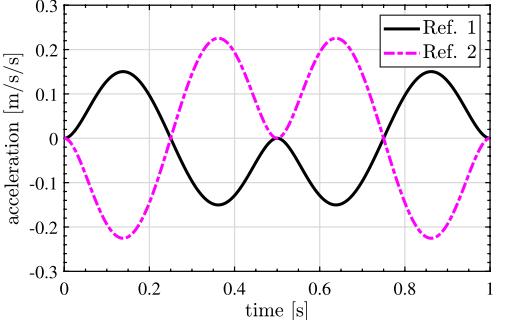

(a) Acceleration.

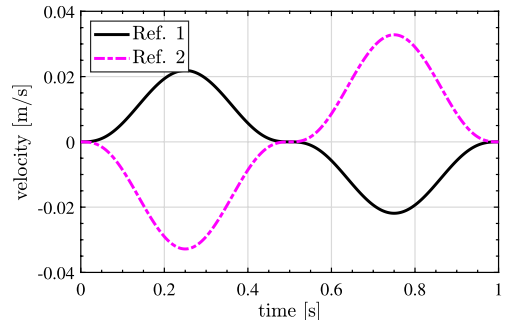

(b) Velocity.

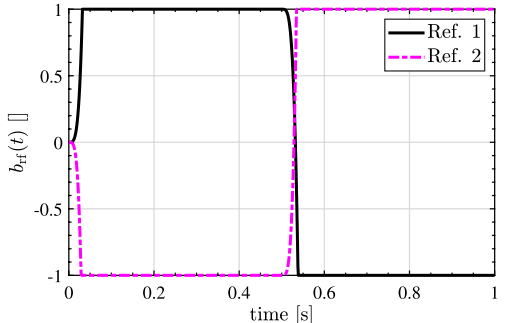

(c) $b_{\mathrm{rf}}(t)$ defined as $(18)\left(x_{\mu}=5 \mu \mathrm{m}\right)$.

Fig. 11. Basis functions of P-ILC for ball-screw-driven stages (Case 1)

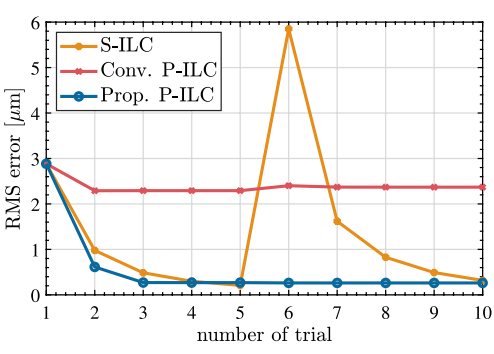

(a) RMS error of each trial.

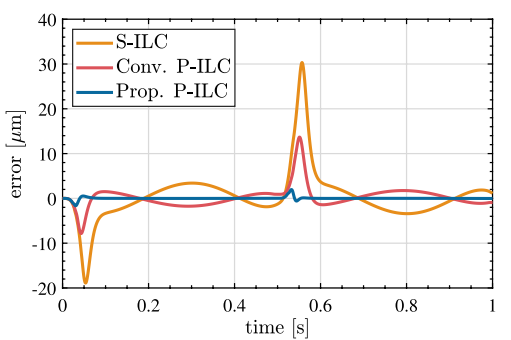

(d) Tracking error of the 6th trial.

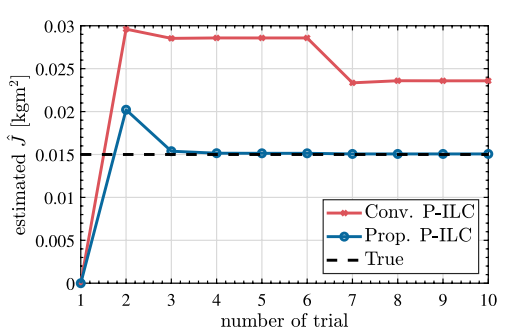

(g) Estimated inertia $\hat{J}$.

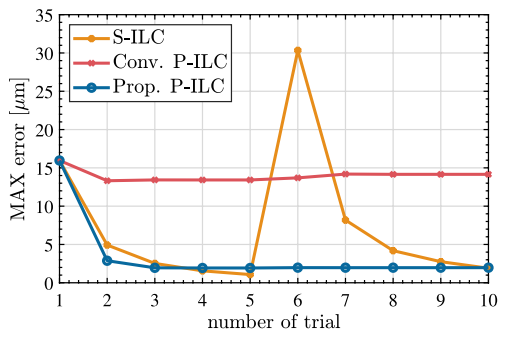

(b) Maximum error of each trial,

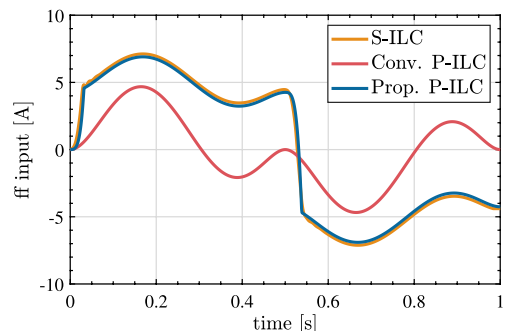

(e) Feedforward input of the 5th trial.

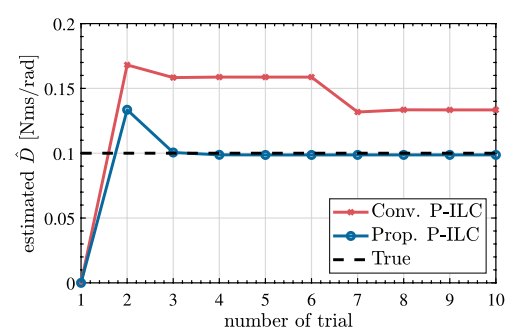

(h) Estimated viscosity coefficient $\hat{D}$.

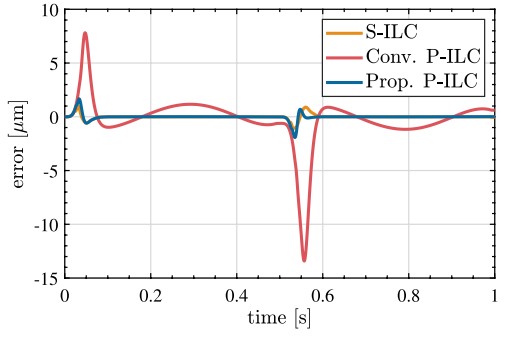

(c) Tracking error of the 5th trial.

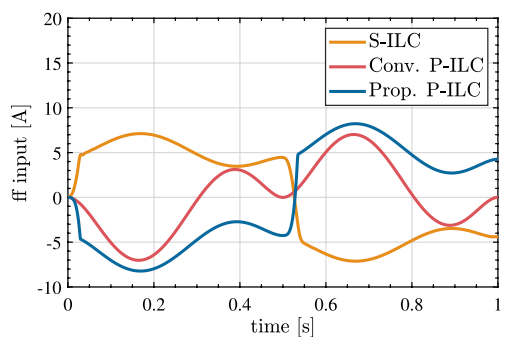

(f) Feedforward input of the 6th trial.

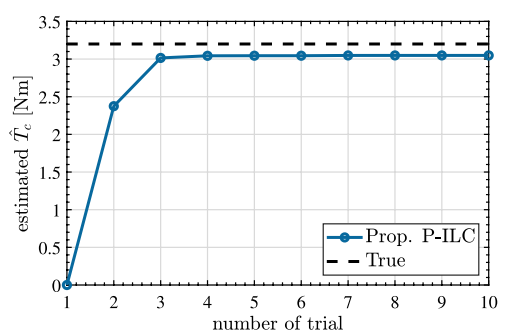

(i) Estimated Coulomb friction $\hat{T}_{c}$.
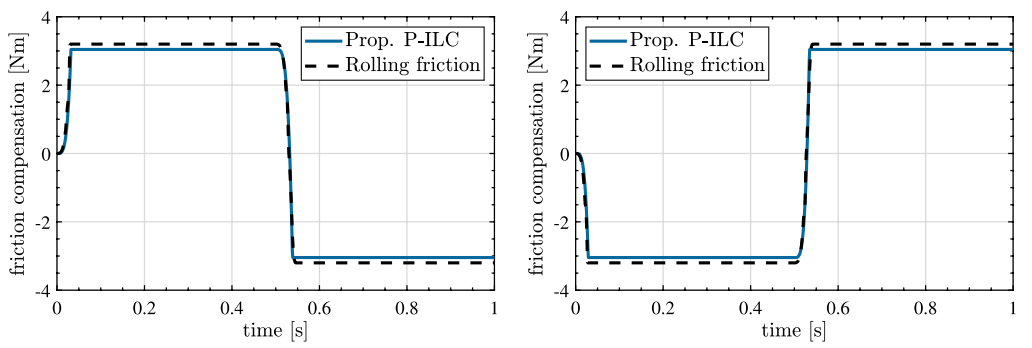

(k) Rolling friction compensation of the 6th trial.

(j) Rolling friction compensation of the 5th trial.

Fig. 12. Simulation results (Case 1)

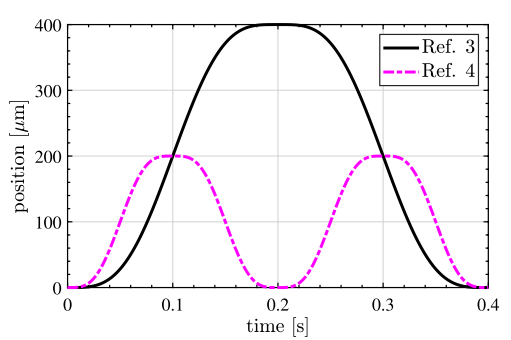

(a) Position References.

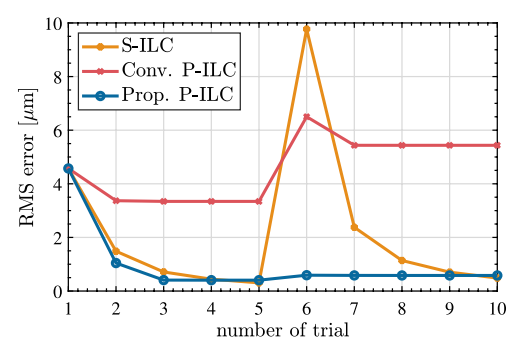

(b) RMS error of each trial.

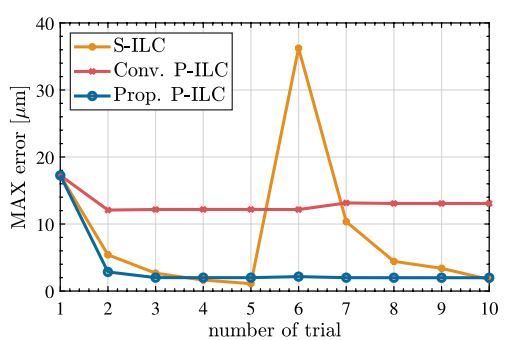

(c) Maximum error of each trial.

Fig. 13. Simulation results (Case 2) 


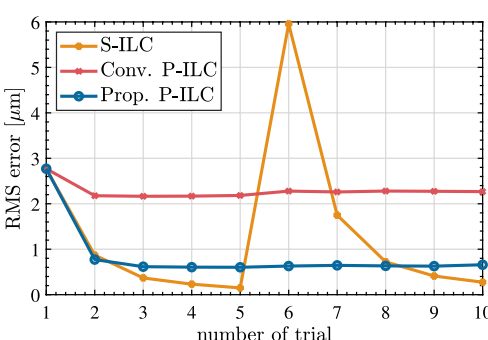

(a) RMS error of each trial

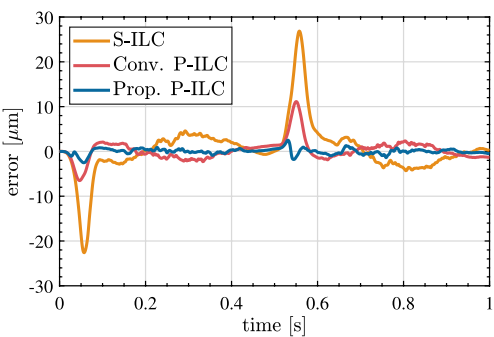

(d) Tracking error of the 6th trial.

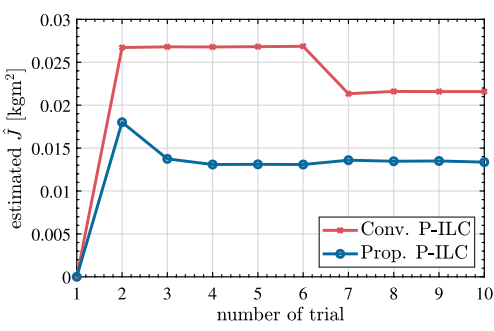

(g) Estimated inertia $\hat{J}$.

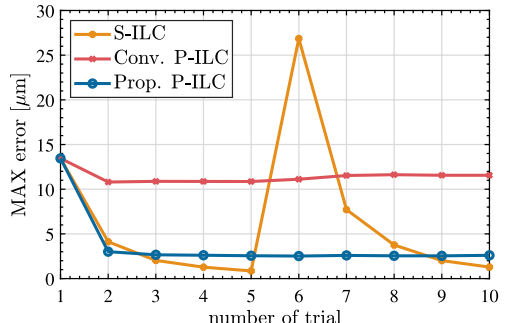

(b) Maximum error of each trial.

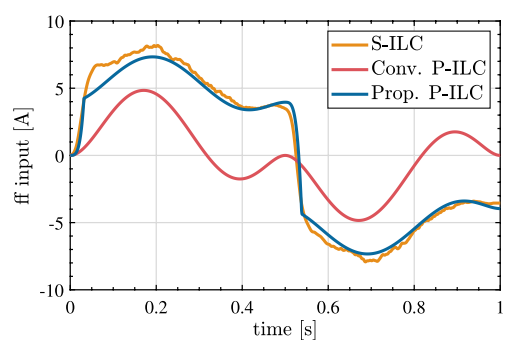

(e) Feedforward input of the 5th trial.

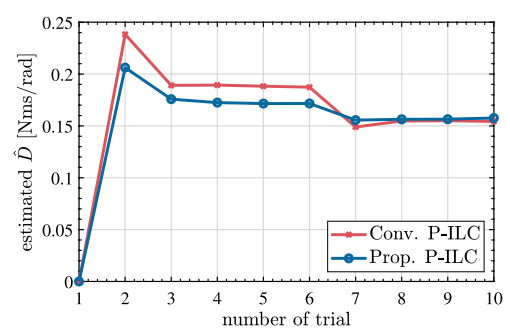

(h) Estimated viscosity coefficient $\hat{D}$.

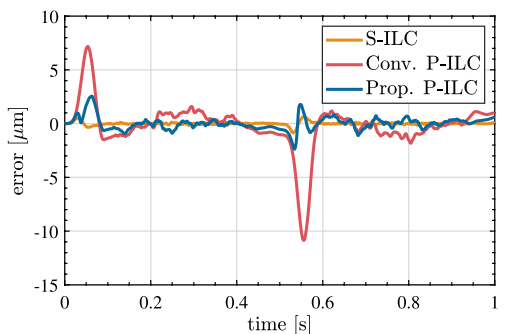

(c) Tracking error of the 5th trial.

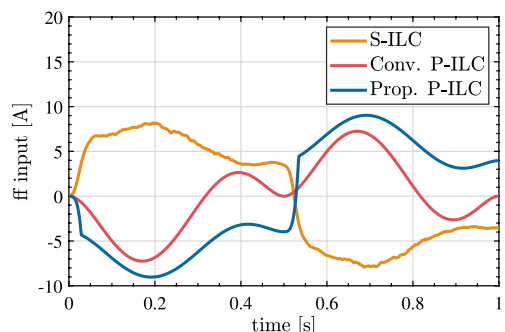

(f) Feedforward input of the 6th trial.

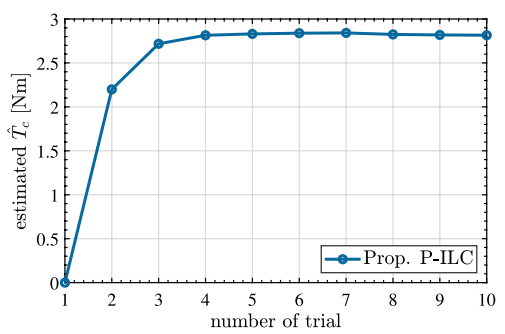

(i) Estimated Coulomb friction $\hat{T}_{c}$.

Fig. 14. Experimental results

ever, the experimental results are worse than the simulation results, see Figs. 12 and 14. When the basis functions are designed, it is assumed that the vibration mode of the ballscrew-driven stages is ignored, and rolling friction shows a constant value in region 2 . These assumptions may deteriorate control performance. Moreover, according to Fig. 14(i), the estimated value of Coulomb friction is smaller than the value measured by ultra-low speed examination. The estimation error also may cause a larger tracking error. To achieve more precise control of ball-screw-driven stages by P-ILC, the choice of basis functions and the estimation method of the plant parameters should be reconsidered.

\section{Conclusion and Future Work}

For precise control of ball-screw-driven stages, rolling friction should be compensated. The compensation methods are classified into two types, model-based and learning-based methods. S-ILC is one of the learning-based methods. However, S-ILC can deal with only precisely repeated tasks and deteriorates when the task is changed. To overcome this problem, studies on P-ILC have been conducted. P-ILC can deal with multiple tasks by using basis functions. However, rolling friction compensation was not considered in previous studies on P-ILC. Therefore, in this study, we propose P-ILC using the basis functions for rolling friction compensation. Simulations and experiments demonstrate that the proposed P-ILC can suppress tracking error significantly compared with the conventional P-ILC without consideration of rolling friction compensation. As future work, modeling of rolling friction, choice of basis functions, and estimation method of the parameters should be improved to achieve precise control of ball-screw-driven stages by P-ILC.

\section{References}

( 1 ) C. Canudas de Wit, H. Olsson, K. Astrom, and P. Lischinsky: "A new model for control of systems with friction", IEEE Transactions on Automatic Control, Vol.40, No.3, pp.419-425 (1995)

( 2 ) F. Al-Bender, V. Lampaert, and J. Swevers: "The Generalized Maxwell-Slip Model: A Novel Model for Friction Simulation and Compensation", IEEE Transactions on Automatic Control, Vol.50, No.11, pp.1883-1887 (2005)

( 3 ) Y. Maeda and M. Iwasaki: "Feedforward Friction Compensation Using the Rolling Friction Model for Micrometer-stroke Point-to-point Positioning Motion”, IEEJ Journal of Industry Applications, Vol.7, No.2, pp.141-149 (2018)

( 4 ) H. Asaumi and H. Fujimoto: "Proposal on nonlinear friction compensation based on variable natural length spring model", in SICE Annual Conference, pp.2393-2398 (2008)

( 5 ) T. Takemura and H. Fujimoto: "Proposal of novel rolling friction compensation with data-based friction model for ball screw driven stage", in IECON 2010 - 36th Annual Conference on IEEE Industrial Electronics Society, Vol.1, pp.1932-1937 (2010)

( 6 ) T. Beauduin and H. Fujimoto: "Distributed and Parameter-Varying Friction Compensation for Ball-Screw Feed Drive Systems", in The 3rd IEEJ International Workshop on Sensing, Actuation, Motion Control, and Optimization, No.6 (2017)

( 7 ) H. Fujimoto and T. Takemura: "High-Precision Control of Ball-ScrewDriven Stage Based on Repetitive Control Using $n$-Times Learning Filter", IEEE Transactions on Industrial Electronics, Vol.61, No.7, pp.3694-3703 (2014)

( 8 ) D.A. Bristow, M. Tharayil, and A.G. Alleyne: "A Survey of Iterative Learning Control”, IEEE Control Systems Magazine, Vol.26, No.3, pp.96-114 (2006)

( 9 ) T. Oomen: "Advanced Motion Control for Precision Mechatronics: Control, Identification, and Learning of Complex Systems", IEEJ Journal of Industry 
Applications, Vol.7, No.2, pp.127-140 (2018)

(10) F. Boeren, A. Bareja, T. Kok, and T. Oomen: "Frequency-Domain ILC Approach for Repeating and Varying Tasks: With Application to Semiconductor Bonding Equipment", IEEE/ASME Transactions on Mechatronics, Vol.21, No.6, pp.2716-2727 (2016)

(11) J. van Zundert, J. Bolder, and T. Oomen: "Optimality and flexibility in Iterative Learning Control for varying tasks”, Automatica, Vol.67, pp.295-302 (2016)

(12) I. Nilkhamhang and A. Sano: "Model-based adaptive friction compensation for accurate position control", in Proceedings of the IEEE Conference on Decision and Control, No.2, pp.1346-1351 (2008)

(13) X. Wang, S. Wang, and B. Yao: "Adaptive robust control of linear electrical loading system with dynamic friction compensation", in 2010 IEEE/ASME International Conference on Advanced Intelligent Mechatronics, pp.908-913 (2010)

(14) F. Sakai: "A Continuous-time System Identification for Systems with Nonlinear Friction Using Iterative Learning Control", Transactions of the Society of Instrument and Control Engineers, Vol.53, No.8, pp.448-454 (2017) (in Japanese)

(15) R. Pintelon and J. Schoukens: System Identification: A Frequency Domain Approach, 2nd ed. Wiley-IEEE Press (2012)

(16) M. Tomizuka: "Zero Phase Error Tracking Algorithm for Digital Control", Journal of Dynamic Systems, Measurement, and Control, Vol.109, No.1, pp.65-68 (1987)

Takumi Hayashi (Student Member) received the B.E. degree from

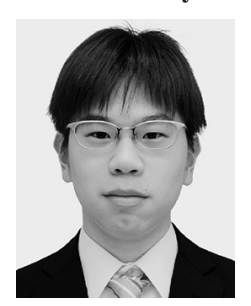
the University of Tokyo, Japan, in 2018. He is currently working towards the M.S. degree in the Department of Advanced Energy, Graduate School of Frontier Sciences, the University of Tokyo. His interests are in control engineering and motion control, especially learning control. He is a student member of IEEE.

Hiroshi Fujimoto (Senior Member) received the Ph.D. degree in the

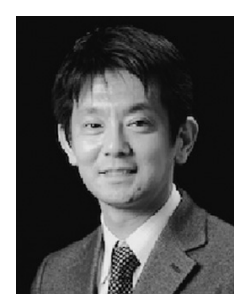

Department of Electrical Engineering from the University of Tokyo in 2001. In 2001, he joined the Department of Electrical Engineering, Nagaoka University of Technology, Niigata, Japan, as a research associate. From 2002 to 2003, he was a visiting scholar in the School of Mechanical Engineering, Purdue University, U.S.A. In 2004, he joined the Department of Electrical and Computer Engineering, Yokohama National University, Yokohama, Japan, as a lecturer and he became an associate professor in 2005 . He is currently an associate professor of the University of Tokyo since 2010. He received the Best Paper Awards from the IEEE Transactions on Industrial Electronics in 2001 and 2013, Isao Takahashi Power Electronics Award in 2010, Best Author Prize of SICE in 2010, the Nagamori Grand Award in 2016, and First Prize Paper Award IEEE Transactions on Power Electronics in 2016. His interests are in control engineering, motion control, nano-scale servo systems, electric vehicle control, motor drive, visual servoing, and wireless motors. He is a senior member of IEEE. He is also a member of the Society of Instrument and Control Engineers, the Robotics Society of Japan, and the Society of Automotive Engineers of Japan. He is an associate editor of IEEE/ASME Transactions on Mechatronics from 2010 to 2014, IEEE Industrial Electronics Magazine from 2006, IEE of Japan Transactions on Industrial Application from 2013, and Transactions on SICE from 2013 to 2016. He is a chairperson of JSAE vehicle electrification committee from 2014 and a past chairperson of IEEE/IES Technical Committee on Motion Control from 2012 to 2013.
Yoshihiro Isaoka (Non-member) joined DMG MORI CO., LTD. in

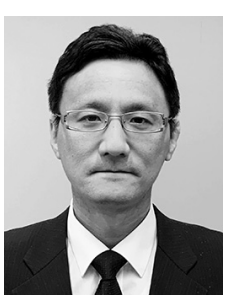
2017, and is presently an assistant chief engineer at Motor Department. He has worked on development of servo control for machine tools.

Yuki Terada (Non-member) joined DMG MORI CO., LTD. in 2008,

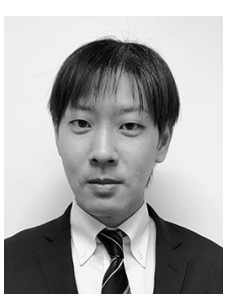
and is presently a Group Manager at Motor Department. He has worked on development of servo control and development of Direct Drive Motor for machine tools. 